\title{
Comparação do desempenho funcional de crianças com visão subnormal e crianças com desenvolvi- mento normal aos 2 e 6 anos de idade*
}

\section{Comparison of performance in children with low vision and children with normal development at 2 and 6 years of age}

\author{
Marisa Cotta Mancini', Marcela Aline Fernandes Braga ${ }^{2}$, \\ Karolina Alves Albuquerque ${ }^{3}$, Thalita Maria Vasconcelos Ramos ${ }^{4}$, \\ Paula Silva de Carvalho Chagas ${ }^{5}$
}

MANCINI, M. C.; BRAGA, M. A. F.; ALBUQUERQUE, K. A.; RAMOS, T. M. V.; CHAGAS, P. S. C. Comparação do desempenho funcional de crianças com visão subnormal e crianças com desenvolvimento normal aos 2 e 6 anos de idade. Rev. Ter. Ocup. Univ. São Paulo, v. 21, n. 3, p. 215-222, set./dez. 2010.

\begin{abstract}
RESUMO: A visão é um sentido fundamental para o ser humano, pois fornece informações diferenciadas de outros sentidos. Existe uma associação entre visão e desenvolvimento, de forma que a redução das capacidades visuais pode comprometer a funcionalidade e a independência, bem como certas áreas do desenvolvimento infantil. O objetivo deste estudo foi comparar a funcionalidade de crianças com visão subnormal e crianças com desenvolvimento normal nas idades de dois e seis anos. Neste estudo transversal foram avaliadas 30 crianças, sendo 14 com dois anos e 16 com seis anos. Elas foram subdivididas em dois grupos, crianças com desenvolvimento normal e crianças com visão subnormal e avaliadas com o teste Inventário de Avaliação Pediátrica de Incapacidade (PEDI). Foi feito equivalência entre os grupos de acordo com o sexo e nível socioeconômico da família. Os resultados sugerem que crianças de dois anos de idade com visão subnormal apresentam menor repertório de habilidade de autocuidado e de mobilidade e necessitam de maior assistência do cuidador, quando comparadas a crianças de mesma idade com desenvolvimento normal. Porém, tais diferenças não se manifestam aos seis anos, já que as crianças com visão subnormal apresentaram desempenho inferior na área de autocuidado, mas não foram observadas diferenças na mobilidade. Os resultados deste estudo contribuem para delinear o perfil funcional de crianças com visão subnormal, indicando as áreas que necessitam ser priorizadas nas ações terapêuticas, direcionando o processo de intervenção.
\end{abstract}

DESCRITORES: Baixa visão; Devenvolvimento humano; Criança.

\footnotetext{
* Estudo realizado como monografia do Curso de Especialização em Terapia Ocupacional - Ênfase em Desenvolvimento Infantil, do Departamento de Terapia Ocupacional, EEFFTO, UFMG. Os dados foram coletados no Departamento de Visão Subnormal do Hospital São Geraldo, do complexo hospitalar do Hospital das Clínicas da Universidade Federal de Minas Gerais.

1. Doutora em Ciências pela Universidade de Boston, Professora Titular do Departamento de Terapia Ocupacional, Escola de Educação Física, Fisioterapia e Terapia Ocupacional (EEFFTO), Universidade Federal de Minas Gerais -UFMG.

2. Especialista em Terapia Ocupacional - ênfase em Desenvolvimento Infantil, DTO, EEFFTO, UFMG.

3. Mestranda em Ciências da Reabilitação, EEFFTO, UFMG.

4. Terapeuta Ocupacional, UFMG.

5. Doutora em Ciências de Reabilitação pela UFMG, Professora Adjunta da Faculdade de Fisioterapia, Universidade Federal de Juiz de Fora.

Endereço para correspondência: Marisa Cotta Mancini, Departamento de Terapia Ocupacional, EEFFTO, Universidade Federal de Minas Gerais, Av. Antonio Carlos, 6627 - Campus Universitário. Pampulha, CEP: 31270-901, e-mail: mcmancini@pib.com.br
} 


\section{INTRODUÇÃO}

Organização Mundial de Saúde (OMS) defi-
ne visão subnormal como sendo a deficiência
visual caracterizada por uma acuidade visual inferior a $6 / 18$, porém igual ou superior a $3 / 60$, ou uma perda de campo visual correspondente a menos de 20 graus no melhor olho, com a melhor correção possível (RESNIKOFF et al., 2004). Ainda assim, a pessoa deve ser capaz, ou potencialmente capaz, de usar a visão para o planejamento e/ou execução de uma tarefa da rotina diária. O termo visão subnormal é muito utilizado na área de saúde, já o termo baixa visão é amplamente empregado na área de educação (RIBEIRO, 2007).

Em 1990, a OMS estimou a existência de 110 milhões de pessoas com deficiência visual grave no mundo, sendo que destes, 38 milhões eram cegos e 72 milhões possuíam visão subnormal ou baixa visão. Segundo estimativas, as crianças com esta condição corresponderiam a $4 \%$ do total, sendo esta condição de saúde em crianças, resultante de diferentes fatores causais (BONATTI et al., 2008). Atualmente, as doenças genéticas, congênitas ou perinatais são reconhecidas como principais causas da deficiência visual da infância, em países desenvolvidos. Já nos países em desenvolvimento, as causas mais comuns incluem as nutricionais e infecciosas. No Brasil, a toxoplasmose e a catarata congênita secundária à rubéola destacam-se como as principais causas da deficiência visual em crianças (HADDAD et al., 2006). A OMS prioriza a prevenção das cegueiras e da baixa visão na infância, por serem determinantes de conseqüências negativas na vida da criança, como baixa expectativa de vida, e por suas repercussões sociais, econômicas e emocionais (HADDAD et al., 2006).

A visão é fundamental para o ser humano, pois fornece informações ambientais que nenhum outro órgão sensorial é capaz de proporcionar. Existe uma associação entre visão e desenvolvimento, de forma que a redução das capacidades visuais pode comprometer a funcionalidade e a independência da criança, em diferentes fases do desenvolvimento, resultando em comprometimentos em diversas áreas do desenvolvimento infantil (FRUTUOSO, 2008). Grande parte das experiências da criança na exploração do ambiente, na descoberta do próprio corpo, na relação com os objetos e com outras pessoas, tem participação direta da visão, que se torna um elemento fundamental na efetivação das ações da criança em seu meio ambiente (SANTOS, 2007).

Diversos aspectos do desenvolvimento infantil, incluindo as capacidades motores, cognitivas e afetivas, podem ser diretamente impactados pela deficiência visual. Acredita-se que tal deficiência pode ainda contribuir com limitações funcionais e possíveis atrasos desenvolvimentais, devido à interação de fatores etiológicos, idade de início, presença de outras deficiências e influências ambientais (HADDAD et al., 2006).

O impacto da deficiência visual nas habilidades funcionais da criança pode acontecer precocemente. Bebês com baixa visão podem apresentar atraso no desenvolvimento motor, pois uma importante fonte de informação que viabiliza a aquisição de novas habilidades apresenta-se comprometida. Lactentes com baixa visão, comparados a bebês com visão normal, podem não encontrar interesse para elevar a cabeça ou engatinhar para alcançar algum objeto (SANTOS, 2007). As crianças que perderam sua visão após os cinco anos de idade, geralmente têm seu potencial visual desenvolvido, conservando memória visual. Porém, crianças que perderam a visão precocemente ou que nasceram cegas, apresentam necessidades de aprendizagem diferentes das demais crianças. O processo de aprendizagem com um sistema visual modificado apresenta ritmo lento, comparado ao mesmo processo em crianças com sistema visual normal. A atitude das pessoas em relação a uma criança com deficiência visual ou baixa visão pode contribuir positiva ou negativamente, inibindo ou estimulando a potencialidade de aprender a interagir nos contextos familiar, escolar e social (MALTA et al., 2006).

As crianças com deficiência visual podem encontrar dificuldades para desempenhar atividades e tarefas da rotina diária. Identificar o impacto funcional da deficiência visual no repertório funcional destas crianças é de fundamental importância para nortear ações terapêuticas. Porém, até o momento, tal informação é escassa na literatura científica. Informações sobre o impacto funcional de condições de saúde que resultam em deficiências, no caso do presente estudo, a visão subnormal, são importantes, pois geralmente, ilustram as queixas e experiências das crianças e/ou de seus familiares, mostrando possíveis metas ou desfechos a serem almejados pelos profissionais da saúde, numa perspectiva da prática centrada no cliente.

Baseando-se nos pressupostos de um serviço centrado no cliente, o atendimento de Terapia Ocupacional deve considerar como cliente, a criança e a família, e buscar promover o processo de funcionalidade. Neste modelo, deve ser garantido à família o direito sobre a escolha nas tomadas de decisões, tratando os pais respeitosamente e fornecendo o suporte necessário, incluindo as informações para lidar na rotina diária, bem como com os sentimentos que a necessidade especial da criança pode gerar nos diversos ambientes que a mesma interage (KING, 1999).

O objetivo deste estudo foi comparar o perfil de funcionalidade, ou seja, o repertório de habilidades funcionais 
de auto-cuidado e de mobilidade, e a independência da criança para realizar tarefas nestas áreas funcionais, de crianças com visão subnormal e crianças com desenvolvimento normal, nas idades de dois e seis anos.

\section{METODOLOGIA}

\section{Participantes}

Participaram desse estudo transversal, 30 crianças da região metropolitana de Belo Horizonte, selecionadas por conveniência, alocadas em dois grupos: 15 crianças diagnosticadas com visão subnormal e sem outros diagnósticos que pudessem causar atraso no desenvolvimento, e outras 15 crianças com desenvolvimento típico, sem distúrbios visuais diagnosticados. Cada um desses dois grupos foi re-dividido em dois subgrupos etários, sendo um deles composto por crianças com idade entre 2 anos e 2 anos e 11 meses, e o outro constituído por crianças com idade entre 6 anos e 6 anos e 11 meses. Assim, as 30 crianças que participaram deste estudo foram alocadas em quatro subgrupos com sete crianças na faixa etária de 2 anos e oito crianças na faixa etária de 6 anos, em cada grupo, respectivamente.

Como critérios de exclusão, as crianças que compuseram a amostra de visão subnormal não poderiam ter qualquer quadro neurológico, cardíaco ou respiratório associado. A amostra foi selecionada em um hospital de referência onde as crianças foram submetidas a avaliações neurológicas, tanto de imagem quanto avaliação clínica, bem como avaliações oftalmológicas para quantificar a visão subnormal segundo os critérios da OMS. Todas as crianças estavam em acompanhamento terapêutico do serviço de Terapia Ocupacional do hospital universitário, em atendimento ambulatorial. $\mathrm{O}$ tamanho da amostra foi determinado pelo esgotamento da população alvo disponível para participar da pesquisa onde a amostra foi selecionada, já que os demais serviços de visão subnormal da cidade não possuíam o mesmo rigor clínico para delineamento do perfil de sua clientela.

As crianças com desenvolvimento normal foram selecionadas em creches e por colegas dos pesquisadores responsáveis pelo estudo. Como critérios de exclusão, as crianças não podiam ter qualquer doença clínica, aguda ou crônica, no momento da participação nesse estudo. As crianças deste grupo foram selecionadas para garantir equivalência de idade, sexo e nível sócio-econômico da família, segundo o Critério de Classificação Econômica Brasil proposto pela Associação Brasileira de Empresas de Pesquisa (ABEP, 2008), com as crianças do grupo de visão subnormal. A classificação da ABEC distingue os grupos de acordo com a posse de itens de bens de consumo doméstico e o grau de instrução do chefe da família. Este critério consiste em um sistema de pontuação padronizada que estima a capacidade de consumo, considerando que a classe sócio-econômica é uma característica familiar. O sistema classifica a população total do questionário em oito classes (A1, A2, B1, B2, C1, C2, D, E) (ABEP, 2008).

As faixas etárias do estudo foram escolhidas levandose em consideração fatores desenvolvimentais. A idade de dois anos foi escolhida, pois nesta faixa etária as crianças que nasceram pré-termo não necessitaram de correção da idade. Sabe-se que durante os dois primeiros anos, os fatores idade gestacional, intercorrências pré, peri e pós-natais, estado nutricional ao nascimento e oferta nutricional, entre outros, podem influenciar o crescimento da criança. Tal influência pode ser observada na avaliação do peso, da estatura e do perímetro cefálico da criança. Além disso, nessa faixa etária ( 2 anos), as crianças estão em processo de grandes mudanças na área de mobilidade (i.e., marcha independente, subir escadas) (MANCINI et al., 2003). Nessa faixa etária, a criança com baixa visão pode apresentar déficits no desenvolvimento motor grosso e fino, pois não há motivação visual para que elas se interessem em explorar o ambiente e os objetos (FRUTUOSO, 2008).

A outra faixa etária escolhida para este estudo foi a de seis anos por ser a idade na qual a criança começa a freqüentar a escola formal e novas habilidades são exigidas nesta fase, incluindo o uso da visão em detalhes para a escrita e atividades motoras finas. Além do possível atraso no desenvolvimento motor conseqüente da baixa visão, podem ocorrer atrasos na noção de permanência do objeto, coordenação visuo-manual, compreensão de tamanho e dimensão de objetos e dificuldade no aprendizado através da manipulação e jogos de construção (SANTOS, 2007).

O presente estudo foi aprovado pelo Comitê de Ética em Pesquisa (parecer ETIC 409-2008). O Termo de Consentimento Livre e Esclarecido foi previamente assinado pelos pais que concordaram participar, com esclarecimentos dos objetivos deste estudo.

\section{Instrumentação e procedimentos}

A versão brasileira adaptada do questionário Pediatric Evaluation of Disability Inventory (Inventário de Avaliação Pediátrica de Incapacidade - PEDI) foi administrada com as mães ou responsáveis pela criança participante do estudo (MANCINI, 2005).

O PEDI é um questionário semi-estruturado utilizado para descrever o desempenho funcional da criança de faixa etária entre 6 meses e 7 anos e meio de idade, nas áreas de autocuidado, mobilidade e função social. O inventário é composto de três partes. A parte I informa sobre as capacidades da criança em realizar atividades funcionais nas três áreas 
citadas; a parte II quantifica a assistência disponibilizada pelo cuidador nas mesmas áreas e a parte III se refere às modificações do ambiente físico utilizadas na rotina diária da criança.

Para esse estudo, foram utilizadas as subescalas de autocuidado e de mobilidade, das partes I e II. Na parte I, os escores atribuídos a cada item informam sobre a capacidade de realização do mesmo, sendo a criança capaz (1) ou não (0) de realizá-lo. Na segunda parte, a pontuação do grau de independência da criança é pontuada inversamente à quantidade de ajuda disponibilizadas pelo cuidador, numa escala ordinal de 5 pontos, sendo 0 (zero) se ela for completamente dependente do cuidador e 5 (cinco) se for independente na realização da tarefa funcional. As pontuações intermediárias, informam sobre níveis diferenciados de ajuda, como por exemplo, supervisão (4) e assistência mínima (3).

O teste foi aplicado somente uma vez, no formato de entrevista com os cuidadores das crianças participantes desse estudo, com duração de 20 a 30 minutos para administração e pontuação da folha de registro do PEDI (MANCINI, 2005). As avaliações foram realizadas nos domicílios das crianças com desenvolvimento normal e no Hospital, no momento que melhor convinha ao responsável pelas crianças com visão subnormal. Dez inventários de crianças com desenvolvimento normal foram respondidos por telefone.

\section{Análise estatística}

Foi realizada análise descritiva dos participantes do estudo, comparando os grupos em relação à idade, sexo, nível socioeconômico (ABEP, 2008) e características diagnósticas. Os dados inferenciais foram analisados utilizando a Análise de Variância (ANOVA), com dois fatores independentes (i.e., idade e grupo) e um fator de interação (i.e., idade x grupo), para comparar as médias dos escores obtidos no teste PEDI, entre os quatro grupos. Uma vez evidenciada diferença significativa no fator de interação, foi utilizado o teste post-hoc Scheffè para localização das diferenças bivariadas. O software Statistical Package for Social Sciences $\left(\right.$ SPSS $^{\circledR}$ ) versão 15.0 foi utilizado, e o nível de significância estabelecido em $\alpha=0,05$.

\section{RESULTADOS}

As características descritivas das crianças participantes do estudo estão disponibilizadas na Tabela 1.

Tabela 1 - Características descritivas das crianças do estudo, referentes a sexo, nível sócio-econômico da família e diagnóstico

\begin{tabular}{|c|c|c|c|c|c|}
\hline & \multirow[b]{3}{*}{ Características } & \multicolumn{2}{|c|}{ Grupo visão subnormal } & \multicolumn{2}{|c|}{ Grupo controle } \\
\hline & & \multicolumn{2}{|c|}{ Grupos etários } & \multicolumn{2}{|c|}{ Grupos etários } \\
\hline & & 2 anos & 6 anos & 2 anos & 6 anos \\
\hline \multirow[t]{2}{*}{ Sexo } & Feminino & 2 & 5 & 2 & 5 \\
\hline & Masculino & 5 & 3 & 5 & 3 \\
\hline \multirow{8}{*}{$\mathrm{NSE}^{\dagger}$} & A1 & - & - & - & - \\
\hline & $\mathrm{A} 2$ & 1 & - & 1 & - \\
\hline & B1 & 1 & - & 1 & - \\
\hline & B2 & 1 & 4 & 1 & 3 \\
\hline & $\mathrm{C} 1$ & 2 & - & - & 1 \\
\hline & $\mathrm{C} 2$ & - & 1 & 1 & 1 \\
\hline & $\mathrm{D}$ & 2 & 3 & 3 & 3 \\
\hline & E & - & - & - & - \\
\hline \multirow{8}{*}{ Diagnóstico $^{\dagger \dagger}$} & Retinopatia da prematuridade & 2 & 3 & & \\
\hline & Hemorragia do vítreo por tocotraumatismo & 1 & & & \\
\hline & Nistagmo/estrabismo & 2 & 1 & & \\
\hline & Catarata Congênita & 1 & 1 & & \\
\hline & Coloboma de disco óptico & - & 1 & & \\
\hline & Baixa acuidade visual cortical & - & 1 & & \\
\hline & Seqüela de toxoplasmose & 1 & - & & \\
\hline & Glaucoma congênito & - & 1 & & \\
\hline
\end{tabular}

${ }^{\dagger}$ Diagnóstico: condições clinicas que possivelmente causaram a visão subnormal. 
MANCINI, M. C.et al. Comparação do desempenho funcional. Rev. Ter. Ocup. Univ. São Paulo, v. 21, n. 3, p. 215-222, set./dez. 2010.

O teste ANOVA evidenciou diferenças nas habilidades funcionais de autocuidado e de mobilidade ( $\mathrm{p} \leq 0,029$ e $p \leq 0,038$, respectivamente), entre os grupos, entre as idades e na interação idade $\mathrm{x}$ grupo. Especificamente no efeito idade, crianças aos dois anos de idade apresentaram desempenho inferior ao de crianças aos seis anos $(\mathrm{p}<$ $0,001)$ nas habilidades de autocuidado e de mobilidade. A interação grupo $\mathrm{x}$ idade, indica que diferenças entre os grupos clínicos são distintas nas duas faixas etárias. Resultados das comparações post-hoc bivariadas revelaram que crianças com visão subnormal apresentam habilidades de autocuidado inferiores às de crianças com desenvolvimento normal $\mathrm{e}$ que a magnitude da diferença entre os dois grupos etários é superior no grupo de visão subnormal do que no de crianças com desenvolvimento normal (Tabela 2 e Figura1).

Nas habilidades funcionais de mobilidade, crianças com dois anos de idade tiveram desempenho inferior ao de crianças típicas, de mesma faixa etária. Aos seis anos de idade essas diferenças desaparecem $(p \geq 0,863)$, sendo o desempenho das crianças com visão subnormal semelhante ao de crianças com desenvolvimento normal aos 2 anos ( $p=$ $0,497)$. Além disso, crianças do grupo visão subnormal de 6 anos apresentam repertório de mobilidade semelhante ao de crianças com desenvolvimento normal, de 2 anos.

Com relação à assistência disponibilizada pelo cuidador na área de autocuidado, crianças com visão subnormal receberam maior ajuda para realização das tarefas desta área, comparadas com as crianças com desenvolvimento normal $(\mathrm{p} \leq 0,008)$. Porém, não houve diferença significativa na interação idade $\mathrm{x}$ grupo $(\mathrm{p}=$ $0,189)$.

A assistência do cuidador na área de mobilidade, revelou diferenças entre os grupos, entre as idades e na interação idade $\mathrm{x}$ grupo $(\mathrm{p} \leq 0,011)$. Resultados das análises post-hoc evidenciaram que crianças com diagnóstico de visão subnormal aos dois anos de idade parecem receber maior ajuda por parte do cuidador do que crianças de dois anos com desenvolvimento normal $(\mathrm{p}<0,001)$. Aos seis anos, as crianças deficientes visuais recebem a mesma quantidade de ajuda oferecida a uma criança típica de dois anos ou de seis anos de idade ( $\mathrm{p}=0,765 \mathrm{e} \mathrm{p}=0,492$, respectivamente). Os escores brutos (média e desvio padrão) obtidos em cada área do teste PEDI investigada nesse estudo, nos quatro sub-grupos estão dispostos na tabela 2 , e a figura 1 apresenta os gráficos ilustrativos dos efeitos de interação idade $\mathrm{x}$ grupo.

Tabela 2 - Média e (desvio padrão) dos escores brutos obtidos no teste PEDI, nas áreas de auto-cuidado e mobilidade, das partes de habilidades funcionais e assistência do cuidador, nos quatro sub-grupos do estudo

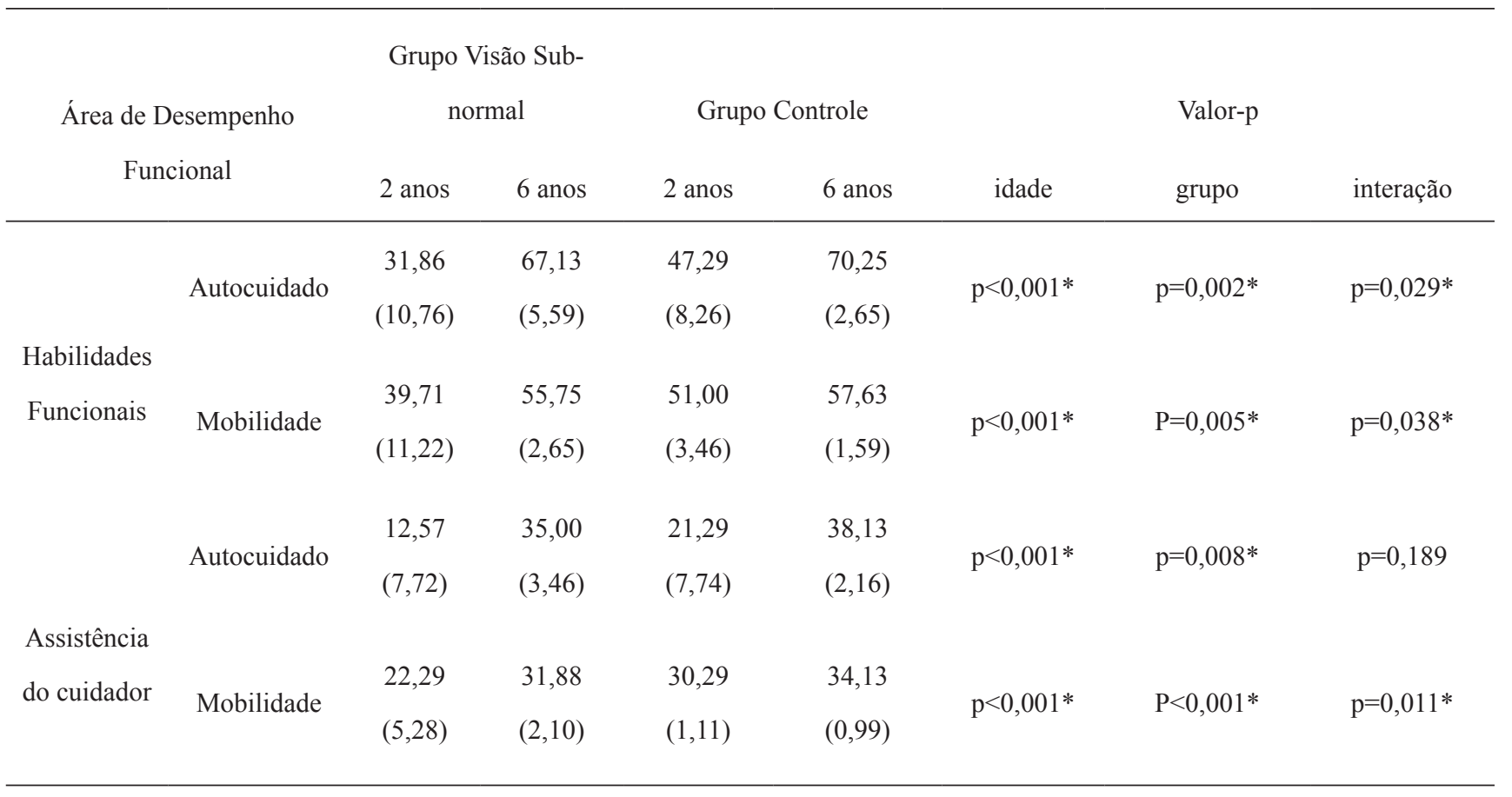

$* \mathrm{p}<0,05$ 
Figura 1 - Gráficos ilustrativos dos efeitos de interação idade $\mathrm{x}$ grupo
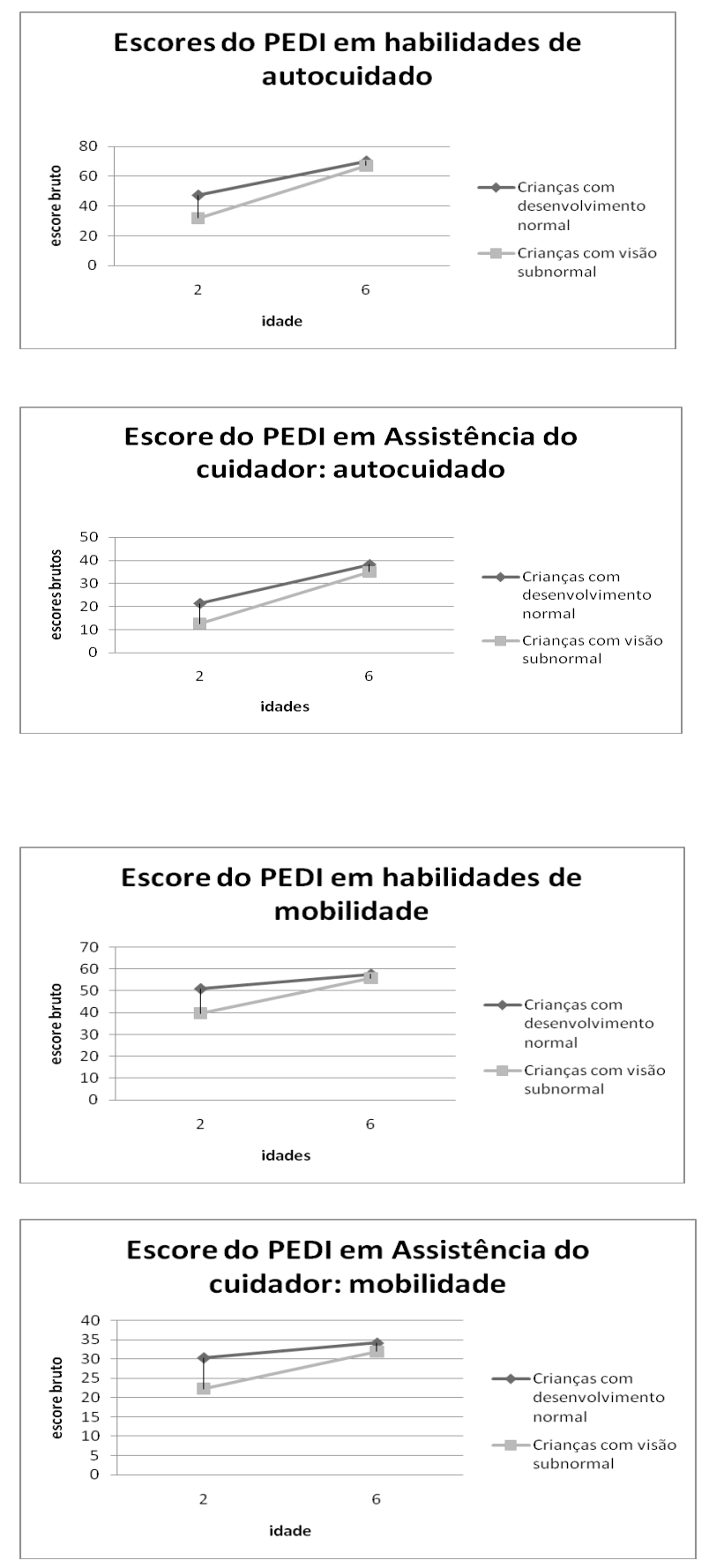

\section{DISCUSSÃO}

Este estudo contribui para a literatura da área de reabilitação, pois descreve o perfil de habilidades funcionais, nas áreas de autocuidado e de mobilidade, bem como a quantidade de assistência disponibilizada pelo cuidador nas duas idades avaliadas, e demonstra diferenças nestas dimensões. Porém, essas diferenças parecem não se manter constantes ao longo do desenvolvimento das crianças com visão subnormal.

No que tange os participantes deste estudo, não houve diferenças entre as crianças dos quatro subgrupos nas características idade e nível socioeconômico das famílias, segundo a ABEP (2008), sugerindo equivalência entre os grupos em variáveis conhecidas na literatura como representando fatores que influenciam no desenvolvimento e desempenho funcional. O diagnóstico mais prevalente entre as crianças com visão subnormal foi a retinopatia da prematuridade, salientando a necessidade de estratégias de intervenção para prevenção da baixa visão infantil (BRITO, 2000).

O desempenho nas habilidades funcionais de autocuidado foi diferente entre crianças típicas e crianças com visão subnormal, tanto aos 2 e quanto aos 6 anos, o que provavelmente ilustra a influência dos déficits visuais na execução destas tarefas (MALTA et al., 2006). Já nas habilidades funcionais de mobilidade, a diferença do desempenho funcional ocorreu somente aos 2 anos de idade. Esta diferença pode ser diretamente relacionada às restrições que a deficiência visual da criança impõe na aquisição de habilidades como localização visual, fixação visual e seguimento visual (PRADO, 2011), as quais são necessárias para a emergência de certas habilidades funcionais como subir meio fio, descer escadas.

A diferença entre grupos observada aos 2 anos não se manteve aos 6 anos. É importante destacar que crianças com visão subnormal participantes deste estudo frequentavam um programa de reabilitação, com a participação de uma terapeuta ocupacional, sendo possível que os esforços terapêuticos tenham influenciado positivamente, disponibilizando oportunidades de experiências visuais e motoras importantes (PRADO, 2011). Portanto, pode-se inferir que as diferenças no desempenho funcional de crianças com visão subnormal e crianças típicas ocorrem de forma não-linear, sendo observadas diferenças em idades iniciais e tais diferenças parecem não se manter na idade pré-escolar. Estes resultados corroboram com evidência demonstrada em um estudo equivalente, realizado com crianças com Síndrome de Down (MANCINI et al., 2003).

O presente estudo acrescenta informações à literatura vigente, já que refuta resultados reportados por Malta et al. (2006), no qual diferenças entre grupos de crianças com visão subnormal e com desenvolvimento típico permaneceram nas diversas idades investigadas (i.e, de 5,8 anos a 7,5 anos). A diferença entre os resultados do presente estudo e de estudo de Malta et al. (2006) pode ser atribuída ao fato de que no 
estudo de 2006 a faixa etária investigada foi mais ampla de tal forma que em intervalos etários maiores, observa-se repertório de habilidades mais variado, e por conseguinte, mudanças funcionais são passíveis de serem evidenciadas. Além disso, apesar da amostra ter sido selecionada em serviços de reabilitação com características aparentemente semelhantes, o rigor na seleção das crianças para o presente estudo parece ter contribuído para os resultados encontrados, tomando-se cuidado para controlar possíveis variáveis de confusão que pudessem interferir nos resultados, tais como a equivalência entre os grupos em relação ao nível socioeconômico da família e a idade das crianças.

A assistência fornecida pelo cuidador na área de mobilidade demonstrou-se diferente nas idades contempladas pelo estudo. Aos dois anos, as crianças com visão subnormal receberam mais ajuda do que as crianças típicas, provavelmente devido à dificuldade ou ausência de capacidade para responder as demandas do ambiente, uma vez que a visão é um fator importante no aprendizado da independência em mobilidade, que muitas vezes se pauta em imitação (OLIVEIRA, 2005). Aos seis anos, as crianças com visão subnormal apresentaram desempenho semelhante ao de crianças típicas, sugerindo que o impacto da condição de visão subnormal é observado em idades anteriores, mas não em idade pré-escolar.

$\mathrm{Na}$ assistência do cuidador em autocuidado, crianças com visão subnormal apresentaram maior dependência para realização das tarefas funcionais, comparadas com as crianças com desenvolvimento normal. Tal resultado ilustra o impacto da visão subnormal na funcionalidade deste grupo clínico, uma vez que estas crianças apresentaram também um repertório de habilidades mais reduzido que as crianças típicas no desempenho de atividades e tarefas da rotina diária, tais como escovar dentes e pentear cabelo. Já aos seis anos de idade, as crianças com visão subnormal receberam maior assistência do cuidador do que as crianças típicas de mesma idade, apesar de apresentarem repertório de habilidades funcionais semelhantes. Tal comportamento por parte dos cuidadores pode demonstrar desconhecimento acerca da dificuldade, superproteção por parte dos cuidadores destas crianças ou subestimação de suas capacidades.

Como relatado no estudo realizado por Figueiredo (2009), as mães desconhecem a dificuldade visual e as reais necessidades dos filhos apesar de freqüentarem serviços especializados em baixa visão. A família e os educadores devem ser orientados sobre as possibilidades de funcionalidade de crianças com visão subnormal, apesar das doenças oculares e deficiências visuais. $\mathrm{Na}$ ausência de habilidades funcionais para desempenhar atividades e tarefas da rotina diária, com independência, formas alternativas ou uso de tecnologia assistiva poderão viabilizar o desempenho funcional de crianças com visão subnormal para atender aos desafios que se apresentam em diferentes contextos e nas diferentes idades (HADDAD et al., 2006).

Como limitação deste estudo, salientamos o pequeno número de participantes. Apesar disso, foi possível encontrar diferenças estatísticas nas variáveis dependentes mensuradas.

As informações disponibilizadas neste estudo são relevantes para profissionais e para serviços de reabilitação infantil que atendem a clientela com deficiência visual. A caracterização do perfil de funcionalidade destas crianças, nas diferentes áreas, pode auxiliar a nortear o planejamento de intervenções e orientações domiciliares, que tenham como objetivo promover a independência funcional da criança no final da primeira infância.

MANCINI, M. C.; BRAGA, M. A. F.; ALBUQUERQUE, K. A.; RAMOS, T. M. V.; CHAGAS, P. S. C. Comparison of performance in children with low vision and children with normal development at 2 and 6 years of age. Rev. Ter. Ocup. Univ. São Paulo, v. 21, n. 3, p. 215-222, set./dez. 2010.

\begin{abstract}
Vision is a fundamental human sense, as it provides distinct information from that of other senses. There is an association between vision and development, so that reduction of visual capabilities may compromise functioning and independence, as well as other areas of child development. The objective of this study was to compare the functioning of children with low vision and normally developing children, of two and six years of age. In this cross-sectional study 30 children were evaluated, 14 with two years and 16 with six years. They were allocated into two groups: a typically developing and a low vision group, and both were assessed with the Pediatric Evaluation of Disability Inventory (PEDI). Group equivalence was attempted for sex and family's socioeconomic status. The results suggest that children with low vision of two years of age have smaller skill repertoire in self-care and mobility, and they need more assistance from their caregiver, when compared to children of same age with normal development. However, these differences were not manifested with children of six years of age, since children with low vision showed limited performance in the area of self-care, but not in mobility. The results from this study contribute to outline the functional profile of children with low vision, indicating areas that may need to be focused in therapeutic actions, helping guide the intervention process.
\end{abstract}

KEY WORDS: Vision, low; Child; Human development. 


\section{REFERÊNCIAS}

ASSOCIAÇÃO BRASILEIRA DE EMPRESAS DE PESQUISA. Critério de classificação econômica do Brasil [Citado em 9 jul. 2008]. Disponível em http://www.abep.org/codigosguias/ Criterio_Brasil_2008.pdf.

BONATTI, F. A. S.; BONATTI, J. A.; SAMPAIO, M. W.; HADDAD, M. A. O.; SOUZA, P. R. M.; JOSÉ, N. K. Avaliação de pacientes utilizando equipamento inovador de auxílio à visão subnormal. Arq. Bras. Oftalmol., v. 71, n. 3, p. 385-388, 2008.

BRITO, P. R.; VEITZMAN, S. Causas de cegueira e baixa visão em crianças. Arq. Bras. Oftalmol., v. 63, n. 1, p. 49-54, 2000.

FIGUEIREDO, M. O.; PAIVA E SILVA, R. B.; NOBRE, M. I. R. Diagnóstico de baixa visão em crianças: sentimentos e compreensão de mães. Arq. Bras. Oftalmol., v. 72, n. 6, p. 766$770,2009$.

FRUTUOSO, A. Deficiência visual na criança. Portal Retina [periódico online], fev. 2008 [Citado 5 jul. 2008]. Disponível em: http://www.portaldaoftalmologia.com.br/home/artigos. asp? cod $=94$.

HADDAD, M. A. O.; CORREA, L. F. J.; WILSON, S. M.; NEWTON, K. J.; Pediatric and adolescent population with visual impairment: study of 385 cases. Clinics, v. 61, n. 3, p. 239-246, 2006.

KING, G.; ROSENBAUM, P.; GOFFIN, R. Family-centered caregiving and well-being of parents of children with disabilities: Linking process with outcome. J. Pediatr. Psychol., v. 24, n. 1, p. 41-53, 1999.

MALTA, J.; ENDRISS, D.; RACHED, S.; MOURA, T.;
VENTURA, L. Desempenho funcional de crianças com deficiência visual, atendidas no Departamento de Estimulação Visual da Fundação Altino Ventura. Arq. Bras. Oftalmol., v. 69, v. 4, p. 571-574, 2006.

MANCINI, M. C. Inventário de avaliação pediátrica de incapacidade (PEDI): manual da versão brasileira adaptada. Belo Horizonte: Ed. UFMG, 2005.

MANCINI, M. C.; SILVA, P. C.; GONÇALVES, S. C.; MARTINS, S. M.; Comparação do desempenho funcional de crianças portadoras de síndrome de Down e crianças com desenvolvimento normal aos 2 e 5 anos de idade. Arq. Neuropsiquiatr., v. 61, n. 2B, p. 409-415, 2003.

OLIVEIRA, J. P.; MARQUES, S. L. Análise da comunicação verbal e não-verbal de crianças com deficiencia visual durante interação com a mãe. Rev. Bras. Educ. Espec., v. 11, n. 3, p. 409-425, 2005.

PRADO, T. F. A. Intervenção visuomotora na Paralisia Cerebral In: CURY, V. C. R.; BRANDÃO, M. B. Reabilitação em paralisia cerebral. Rio de Janeiro: Medbook, 2011. p. 81-93.

RESNIKOFF, S.; PASCOLINI, D.; ETYA'ALE, D; KOCUR, I.; PARARAJASEGARAM, R.; POKHAREL, G. P.; et al. Global data on visual impairment in the year 2002. Bull World Health Organ., v. 82, n. 11, p. 844-851, 2004.

RIBEIRO, L. B. Disfunção visual. In: CAVALCANTI, A.; GALVÃO, C. Terapia ocupacional: fundamentação e prática. Rio de Janeiro: Guanabara Koogan, 2007. p. 399-416.

SANTOS, L. C.; PASSOS, J. E. O. S.; REZENDE, A. L. G.; Os efeitos da aprendizagem psicomotora no controle das atividades de locomoção sobre obstáculos em crianças com deficiência da visão. Rev. Bras. Educ. Espec., v. 13, n. 3, p. 365-380, 2007. 